\title{
The Lamb Shift of Excited $S$-Levels in Hydrogen and Deuterium Atoms
}

\author{
Savely G. Karshenboim ${ }^{1,2}$ \\ D. I. Mendeleev Institute for Metrology (VNIIM) \\ St. Petersburg 198005, Russia
}

\footnotetext{
${ }^{1}$ E-mail: sgk@onti.vniim.spb.su; karshenboim@phim.niif.spb.su

${ }^{2}$ The summer address: Max-Planck-Institut für Quantenoptik, 85748 Garching, Germany. E-mail: krp1@mpq.mpg.de
} 


\begin{abstract}
A specific combination of $s$-state Lamb shift $\Delta E_{L}\left(1 s_{1 / 2}\right)-n^{3} \Delta E_{L}\left(n s_{1 / 2}\right)$ is considered. Its value in calculated both in the hydrogen and deuterium atoms for $n$ up to 12 . The result inludes all correction which can contribute $1 \mathrm{kHz}$ and particulary: one-loop self energy and vacuum polarization, twoloop contribution. Nuclear finite-size corrections for the isotopic difference of the combination are also evaluated.
\end{abstract}




\section{Introduction}

Recently new experimental results on the hydrogen and deuterium Lamb shift have been obtained [1]-[5] and some even higher-precision experiments are going to be completed [6, 7]. The each measurement data include a combination of the Lamb shift of several $n s_{1 / 2}$ levels $(n=1 \div 12)$. To obtain correlations between theoretical calculations of the Lamb shift of $s$-states with different values of principal quantum number $n$ or their combinations, the specific difference of Lamb shifts

$$
\Delta(n)=\Delta E_{L}\left(1 s_{1 / 2}\right)-n^{3} \Delta E_{L}\left(n s_{1 / 2}\right)
$$

is considered in this work.

The values $\Delta(n)$ have to be used to find self-consistent values of the ground state Lamb shift and the Rydberg constant [8, 9].

\section{General expression and definitions}

The Lamb shift is defined here as a shift from the value of level energy

$$
E\left(n l_{j}\right)=m_{r}[f(n j)-1]-\frac{m_{r}^{2}}{2(M+m)}[f(n j)-1]^{2}
$$

where

$$
f(n j)=\left(1+\frac{(Z \alpha)^{2}}{\left[n-j-1 / 2+\sqrt{(j+1 / 2)^{2}-(Z \alpha)^{2}}\right]^{2}}\right)^{-1 / 2}
$$

is the dimensionless Dirac energy with infinite nuclear mass, $m_{r}$ is the reduced mass, $Z$ is the nuclear charge in units of the proton one, and relativistic units in which $\hbar=c=1$ and $\alpha=e^{2}$ are used.

The general expression for eq.(11) has the form [8, 9]

$$
\Delta(n)=\frac{\alpha(Z \alpha)^{4}}{\pi} \frac{m_{r}^{3}}{m^{2}} \times\left\{-\frac{4}{3} \log \frac{k_{0}(1 s)}{k_{0}(n s)}\left(1+Z \frac{m}{M}\right)^{2}+(Z \alpha)^{2} \times\right.
$$




$$
\begin{aligned}
& {\left[\left(4(\log n-\psi(n+1)+\psi(2))-\frac{77\left(n^{2}-1\right)}{45 n^{2}}\right) \log \frac{1}{(Z \alpha)^{2}}+A_{60}^{V P}(n)+G_{n}^{S E}(Z \alpha)\right]} \\
& \left.-\frac{14}{3} \frac{Z m}{M}\left(\psi(n+1)-\psi(2)-\log n+\frac{n-1}{2 n}\right)\right\}+\frac{\alpha^{2}(Z \alpha)^{6} m}{\pi^{2}} \log ^{2} \frac{1}{(Z \alpha)^{2}} B_{62},
\end{aligned}
$$

where $\psi(z)=(d / d z) \log \Gamma(z)$.

Here

- $\log k_{0}(n s)$ is the Bethe logarithm, its value can be found in Refs. [10, 11];

- a logarithmic coefficient in the term $\alpha(Z \alpha)^{6} \log (Z \alpha) m$ was obtained in Ref. [12];

- $G_{n}^{S E}(Z \alpha)$ is one-loop self-energy correction in the order of $\alpha(Z \alpha)^{6} m$ and higher;

- $A_{60}^{V P}(n)$ is the $\alpha(Z \alpha)^{6} m$-contribution of the vacuum polarization;

- $B_{62}$ is the leading logarithmic two-loop correction coefficient;

- the three-loop term of the order $\alpha^{3}(Z \alpha)^{4} m$ in eq.(3) is known and equal to zero;

- recoil corrections in orders $(Z \alpha)^{4} m^{2} / M$ and $(Z \alpha)^{6} m^{3} / M^{2}$ are equal to zero;

- the nuclear finite-size contributions for the difference (3) are small and not included.

The Bethe logarithm is presented in Table 1. The one- and two-loop contributions and recoil corrections are discussed below. The nuclear charge distribution correction is considered in appendix B. 


\section{One-loop self-energy contribution}

\subsection{Extrapolation over $Z$}

The values of $G_{n}^{S E}(Z \alpha)$ with $n=2 \div 5$ for the hydrogen atom can be derived from the numerical data given in Refs. [13, 14] after extrapolation to $Z=1$, with including for the difference ([1]) only two terms [8, 9]

$$
G_{n}^{S E}(Z \alpha)=A_{60}^{S E}(n)+(Z \alpha) A_{70}^{S E}(n)
$$

because the logarithmic coefficient in the order $\alpha(Z \alpha)^{7} m$ has the form [8]

$$
A_{71}^{S E}\left(n l_{j}\right)=\pi\left(\frac{139}{64}-\log 2\right) \delta_{l 0}
$$

and does not contribute to the difference $\Delta(n)$. In case of $n=2$ a result from Ref. 15] for $A_{60}^{S E}(2)$ has been also used.

The input data and the results of the extrapolation are given in Table 2.

The quoted uncertainties arise from statistical errors of the numerical integrations of Refs. [13, 14] and from estimates of the systematic error of the fit of eq.(4).

\subsection{Extrapolation over $n$}

The extrapolated value of $G_{n}^{S E}(\alpha)$ can be rewritten in the form

$$
G_{n}^{S E}(\alpha)=\frac{n-1}{n^{2}} \widetilde{G}_{n}
$$

which is more convenient for the extrapolation over $n$. This transformation is needed because the $n$-dependence of $G_{n}^{S E}(\alpha)$ is not well-behaved. That is easy to see from the trivial result

$$
G_{1}^{S E}(\alpha)=0
$$

After extracting the factor $(n-1)$ explicitly the dependence of $\widetilde{G}_{n}$ over $n$ became quite well-behaved, as well as in case of other coeffitients of eq.(3).

Our results for $n=6 \div 12$ are presented in Table 3 . 


\section{One-loop vacuum polarization}

As it was demonstrated in Ref. [16, for a calculation of the contribution to the difference $\Delta(n)$ it is sufficient to use the approximate wave function

$$
\psi=\left(\begin{array}{c}
1 \\
\frac{\mathbf{p} \sigma}{2 m}
\end{array}\right) \varphi
$$

where $\varphi$ is the Schrödinger wave function, and the effective local potential

$$
V_{V P}(\mathbf{r})=\frac{\alpha(Z \alpha)}{m^{2}}\left(-\frac{4}{15} \delta(\mathbf{r})-\frac{1}{35} \frac{\nabla^{2}}{m^{2}} \delta(\mathbf{r})\right) .
$$

The difference coefficient of the vacuum polarization contribution is found to be 16]

$$
A_{60}^{V P}(n)=\frac{4}{15}\left\{\log n-\psi(n+1)+\psi(2)+\frac{n^{2}-1}{28 n^{2}}+\frac{2(n-1)}{n^{2}}\right\} .
$$

Results for $n=2$ and $n=4$ are in agreement with those from Refs. [17 and [18]. The approximation of eqs. (6, 7) leads to the same result as in work [19] for $n p_{j}$-states.

\section{Two-loop corrections}

The leading two-loop contribution has order $\alpha^{2}(Z \alpha)^{6} m \log ^{2}(Z \alpha)$. It originates from two-loop self energy of an electron in the Coulomb field [8, 9], a general expression of which has the form [22]

$$
\begin{gathered}
\delta E_{L}^{(2)}\left(n l_{j}\right)=\left\langle n l_{j}\left|\Sigma_{1}\left(E_{n l_{j}}\right) \bar{G}_{C}\left(E_{n l_{j}}\right) \Sigma_{1}\left(E_{n l_{j}}\right)\right| n l_{j}\right\rangle \\
+\left\langle n l_{j}\left|\Sigma_{2}\left(E_{n l_{j}}\right)\right| n l_{j}\right\rangle+\left.\left\langle n l_{j}\left|\Sigma_{1}\left(E_{n l_{j}}\right)\right| n l_{j}\right\rangle\left\langle n l_{j}\left|\frac{\partial \Sigma_{1}(E)}{\partial E}\right| n l_{j}\right\rangle\right|_{E=E_{n l_{j}}},
\end{gathered}
$$

where $\Sigma_{r}(E)$ is the $r$-loop one-particle-irreducible self-energy operator of an electron in the Coulomb field and $\bar{G}_{C}(E)$ is the reduced Coulomb Greens function. 
In the Yennie gauge [20, 21], in which the photon propagator in momentum space has the form

$$
D_{\mu \nu}^{Y}(k)=\frac{1}{k^{2}}\left(g_{\mu \nu}+2 \frac{k_{\mu} k_{\nu}}{k^{2}}\right),
$$

the result arises from only the first item of eq.(9) and the ladder part of the second one. Contributions of separated diagrams to different energy levels are presented in Table 4.

The contribution was found in Refs. [8, 24, 26, 25] and finally it has the form

$$
B_{62}=\frac{16}{9}\left(\log n-\psi(n)+\psi(1)-\frac{n-1}{n}+\frac{n^{2}-1}{4 n^{2}}\right) .
$$

The uncertainty of two-loop correction is due to terms beyond $\log ^{2} Z \alpha$ and it is estimated as half the contribution of the leading logarithmic term.

The same diagrams contribute in the order $\alpha^{2}(Z \alpha)^{6} m \log Z \alpha$ also to the decay widths of $n s$ - and $n p$-levels (see for details Ref. [30]), which can be presented as the imaginary part of the self-energy of an electron. This fact is useful for checking [25]. An expecit expression of $\bar{G}_{C}\left(0, \mathbf{r} ; E_{n l}\right)$ found in Ref. 30 has also been used for that.

\section{Recoil corrections}

The evaluation of pure recoil contributions has recently been completed for $s$-levels in the orders $(Z \alpha)^{6} m^{2} / M$ 31, 32 and $(Z \alpha)^{4} m^{3} / M^{2}$ 33.

The results for the term of order $(Z \alpha)^{6} \mathrm{~m}^{2} / M$

$$
\delta E(n s)=\frac{(Z \alpha)^{6} m^{2}}{n^{3} M}\left(4 \ln 2-\frac{7}{2}\right), \quad[31],
$$

and

$$
\delta E(n s)=\frac{(Z \alpha)^{6} m^{2}}{n^{3} M}\left(4 \ln 2-\frac{5}{2}\right), \quad[32],
$$

are in disagreement. In our definitions (2) both of them do not contribute to the difference $\Delta(n)$. Numerical results for recoil correction in the $1 s$ - and 
$2 s$-states obtained in Ref. [34] without any expanssion over $(Z \alpha)$ confirm the scaling factor $1 / n^{3}$ (see table 5) and they are in fair agreement with eq.(11). It should be also menshioned that results of Yelkhovsky [32] and Pachucki [35] for higher- $l$ levels and in agreement one with the other, and with the analytic result for $p$-state of Ref. [36] and the numerical ones for $2 p_{1 / 2}$ and $2 p_{3 / 2}$ of Refs. [34] and [37], respectively.

The correction in order $(Z \alpha)^{4} m^{3} / M^{2}$ contributes only for nuclear spin $I=0$ or 1 , but not for $I=1 / 2$ 33]. The result is

$$
\delta E(I=0,1)=-\frac{1}{2} \frac{m^{3}}{M^{2}} \frac{(Z \alpha)^{4}}{n^{3}} \delta_{l 0} .
$$

All recoil corrections in this section are equal to zero for the difference (1), but no proof on these cancelations is not known without direct calculations.

\section{Results}

The differences $\Delta(n)$ and the contributions to it for the lowest $n s$-levels of hydrogen and deuterium are presented in Table 6 .

The uncertainty of the theoretical expression is a rms sum of the uncalculated terms of orders $\alpha^{2}(Z \alpha)^{6} m \log (Z \alpha)$ and $\alpha^{2}(Z \alpha)^{6} m$, which are estimated as half the contribution of the leading logarithmic term and the uncertainty of the extrapolation for the one-loop self-energy contribution. The uncertainty of the isotopic difference

$$
\Delta^{I s o}(n)=\Delta^{D e u}(n)-\Delta^{H y d}(n)
$$

is neglegible and the nuclear finite-size corrections could be important for it. They are considered in appendix B and included in the results in Table 6.

\section{ACKNOWLEDGEMENTS}

The author would like to thank T. W. Hänsch, A. Nekipelov, K. Pachucki, T. Udem, V. Shabaev, M. Weitz and A. Yelkhovsky for stimulating discussions and the authors of Refs. 3, 4, 5, 6, 7, 18, 29, 32, 34, 37 for informing him on their results prior the publication. The final part of this work was done 
durinig the authors summer stay at the Max-Planck-Institut für Quantenoptik and he is very grateful them for hospitality.

This work was supported in part by grant \# 95-02-03977 of the Russian Foundation for Basic Research. The part of the work was done by collaboration with V. Ivanov and presented at the CPEM96, and the author acknoledges him for the cooperation and their organizing commitee for support of the poster. The author thanks the $28^{\text {th }}$ EGAS Organizing Commitee for support of his participation a the conference, where this work was also presented. 


\section{A Definitions}

The definition of the Lamb shift which is used in this work is due to a shift from the energy level given in eq.(国). It is denoted in this Appendix as $E^{I}(n j)$.

The expansion of $E(n j)$ has the form

$$
E=m \sum_{0}^{\infty}\left(\frac{m}{M}\right)^{a}(Z \alpha)^{b} E_{a b}
$$

An other definition which is also often used include only all $E_{0 b}$ (i. e. the exact Dirac energy with the infinite nuclear mass) terms and $E_{a 2}$ (the Schrödinger energy with reduced mass) and a relativistic recoil term $E_{14}$ :

$$
E^{I I}(n j)=m f(n j)+\left[m_{R}-m\right]\left(-\frac{(Z \alpha)^{2}}{2 n^{2}}\right)+\frac{(Z \alpha)^{4}}{2 n^{3}} \frac{m}{M}\left[\frac{1}{j+1 / 2}-\frac{1}{n}\right] .
$$

Hence the main exluded items in value $E^{I I}$ are

$$
E_{16}=-\frac{1}{2}\left[-\frac{2}{n^{3}(2 j+1)^{3}}-\frac{3}{n^{4}(2 j+1)^{2}}+\frac{4}{n^{5}(2 j+1)}-\frac{1}{n^{6}}\right]
$$

and

$$
E_{24}=\frac{1}{2 n^{3}}\left[-\frac{1}{j+1 / 2}+\frac{3}{2 n}\right] .
$$

Advantages of the first definition of eq.(2) are: extra $E_{16}$ terms do not contribute to $\Delta(n)$ and to the fine structure.Also extra $E_{24}$ terms do not contribute to $E(n s)$ (for nuclear spin $=1 / 2$ ) and to the difference $\Delta(n)$ for any nuclear spin.

Difference between definitions of eq.(2) and eq.(14) in case of the hydrogen atom leads to the shifts presented in Table 7 .

\section{B Nuclear charge distribution correction}

Using the nonrelativistic approximation (the same as in case of vacuum polarisation) the nuclear charge distribution correction to the difference can easy be found to be (cf. [16]) 


$$
\delta \Delta(n)=\mathcal{E}(1 s) \times(Z \alpha)^{2}\left[\psi(n+1)-\psi(2)-\log n-\frac{(n-1)(n+9)}{4 n^{2}}\right],
$$

where $\mathcal{E}(1 s)$ is the well-known nuclear charge distribution correction to the energy of the $1 s$ state

$$
\mathcal{E}(1 s)=\frac{2}{3}(Z \alpha)^{4} m_{R}^{3}\left\langle r^{2}\right\rangle
$$

One can see that the correction to difference is small and can be neglected both for hydrogen and deuterium atom, but for isotopic difference. They are slightly $n$-dependent (cf. term $\Delta^{V P}(n)$ in table 6) and the contribution to $\Delta(n)(n=2-8)$ can be estimated as $-0.049(9) k H z$ for hydrogen, $-0.318(33) \mathrm{kHz}$ for deuterium and as $-0.269(32) \mathrm{kHz}$ for the isotopic difference. The uncertainty takes into acount both: $n$-dependence and the disagreements in proton [38, 39, 40] and deuteron 44, 42] radii measurements. 


\section{References}

[1] F. Schmidt-Kaler, D. Leibfried, M. Weitz and T. W. Hänsch, Phys. Rev. Lett. 70, 2261 (1993).

[2] M. Weitz, A. Huber A. F. Schmidt-Kaler, D. Leibfried and T. W. Hänsch, Phys. Rev. Lett. 72, 328 (1994).

[3] M. Weitz, A. Huber A. F. Schmidt-Kaler, D. Leibfried, W. Vassen, C. Zimmermann, K. Pachucki, T. W. Hänsch, L. Julien and F. Biraben, Phys. Rev. 75, 2664 (1995).

[4] D. J. Berkeland, E. A. Hinds and M. G. Boshier, Phys. Rev. Lett. 75, 2470 (1995).

[5] S. Bourzeix, B. de Beauvoir, F. Nez, M. D. Plimmer, F. de Tomasi, L. Julien and F. Biraben, Phys. Rev. Lett. 76, 384 (1996).

[6] T. W. Hänsch, privat communication.

[7] F. Biraben, privat communication.

[8] S. G. Karshenboim, ZhETF 106, 414 (1994) /in Russian/; JETP 79, 230 (1994).

[9] S. G. Karshenboim, Yad. Fiz. 58, 309 (1995)/in Russian/; Phys. At. Nucl. 58, 262 (1995).

[10] G. W. F. Drake and R. A. Swainson, Phys. Rev. A41 (1990) 1243.

[11] S. Klarsfeld and A. Maquet, Phys. Lett. B43, 201 (1973).

[12] G. W. Erickson and D. R.Yennie, Ann. Phys. 35, 271 (1965); ibid., 447.

[13] P. J. Mohr and Y.-K. Kim, Phys. Rev. A45, 2727 (1992).

[14] P. J. Mohr, Phys. Rev. A46, 4421 (1992).

[15] K. Pachucki, Ann. Phys. 226, 1 (1993). 
[16] V. G. Ivanov and S. G. Karshenboim, 1996 Conference on Precision Electromagnetic Measurements. Conference digest, 636. Braunschweig, 1996; Yad. Fiz. (1996) /in Russian/; Phys. At. Nucl. (1996), to be published.

[17] P. J. Mohr, Phys. Rev. Lett. 34, 1050 (1975); in Beam-Foil Spectroscopy, ed. by I. A. Sellin and D. J. Pegg (Plenum Press, New York, 1976), Vol. 1, p. 89; At. Data Nucl. Data Tables 29, 453 (1983).

[18] K. Pachucki, D. Leibfried, M. Weitz, A. Huber, W. König and T. W. Hänsch, J. Phys. B29, 177 (1996); ibid., 1573.

[19] N. L. Manakov, A. A. Nekipelov and A. G. Fainshtein, ZhETF 95, 1167 (1989)/in Russian/; Sov. Phys, JETP 68, 673 (1989).

[20] A. A. Abrikosov, ZhETF 30, 96 (1956) /in Russian/; Sov. Phys. JETP 3 (1956) 71 ;

L. D. Landau, A. A. Abrikosov, and I. M. Khalatnikov, Nuovo Cim. Suppl. 3, 80 (1956).

[21] H. M. Fried and D. R. Yennie, Phys. Rev. 112, 1391 (1958).

[22] R. Mills and N. Kroll, Phys. Rev. 98, 1489 (1955).

[23] S. G. Karshenboim, ZhETF 103, 1105 (1993) /in Russian/; JETP 76, 541 (1993).

[24] S. G. Karshenboim, Yad. Fiz. 58, 707 (1995) /in Russian/; Phys. At. Nucl. 58, 649 (1995).

[25] S. G. Karshenboim, ZhETF 109, 752 (1996) /in Russian/; JETP 82, 403 (1996).

[26] S. G. Karshenboim, J. Phys. B29, L21 (1996).

[27] S. G. Karshenboim, Yad. Fiz. 58, 901 (1995) /in Russian/; Phys. At. Nucl. 58, 835 (1995).

[28] S. G. Karshenboim, ZhETF 108, 1061 (1995) /in Russian/; JETP 81, 593 (1995). 
[29] K. Pachucki, privat communication.

[30] V. G. Ivanov and S. G. Karshenboim, ZhETF 109, 1219 (1996)/in Russian/; JETP 82, 656 (1996); Phys. Lett. A210, 313 (1996).

[31] K. Pachucki and H. Grotch, Phys. Rev. A51, 1854 (1995).

[32] A. S. Yelkhovsky, preprint BudkerINP-95-63; hep-ph/9508284, ZhETF (1996) /in Russian/; JETP (1996), to be published.

[33] K. Pachucki and S. G. Karshenboim, J. Phys. B28, L221 (1995).

[34] A. N. Artemyev, V. M. Shabaev and V. A. Yerokhin, Phys. Rev. A 52, 1884 (1995).

[35] K. Pachucki, Laser Spectroscopy. XII International Conference (World Sci., Singapore. New Jersey.), ed., M. Inguscio, M. Allegrini, A. Sasso. P. 103.

[36] E. A. Golosov, I. B. Khriplovich, A. I. Milstein and A. S. Yelkhovsky, ZhETF 107, 393 (1995) /in Rusian/; JETP 80, 208 (1995).

[37] A. N. Artemyev, V. M. Shabaev and V. A. Yerokhin, J. Phys. B 28, $5201(1995)$.

[38] L. Hand, D. I. Miller and R. Willson, Rev. Mod. Phys. 35, 335 (1963).

[39] G. G. Simon, Ch. Schmitt et al. Nucl. Phys. A333, 381 (1980).

[40] P. Mergell, U.G. Meissner and D. Drechsel, Nucl. Phys. A596, 367 (1996).

[41] S. Klarsfeld et al, Nucl. Phys. A456, 373 (1986).

[42] J. L. Friar, G. L. Payne, V. G. J. Stoks and J. J. de Swart, Phys. Lett. B311, 4 (1993). 


\begin{tabular}{|c|c|c|}
\hline Level & $\log k_{0}(n l)$ & Ref. \\
\hline $1 s$ & 2.9841285558 & 11 \\
\hline $2 s$ & 2.8117698931 & $11]$ \\
\hline $3 s$ & 2.7676636125 & 11 \\
\hline $4 s$ & 2.7498118405 & 11 \\
\hline $5 s$ & 2.7408237279 & 10 \\
\hline $6 s$ & 2.7356642069 & 10 \\
\hline $7 s$ & 2.7324291292 & 10 \\
\hline $8 s$ & 2.7302672607 & 10 \\
\hline $9 s$ & 2.7287511660 & 10 \\
\hline $10 s$ & 2.7276469387 & 10 \\
\hline $11 s$ & 2.7268177825 & 10 \\
\hline $12 s$ & 2.7261793406 & 10 \\
\hline
\end{tabular}

Table 1: Bethe logarithms. 


\begin{tabular}{|c|c|c|c|c|}
\hline \multicolumn{5}{|c|}{ Input data for the extrapolation over $Z$} \\
\hline$Z$ & $G_{2}^{S E}(Z \alpha)$ & $G_{3}^{S E}(Z \alpha)$ & $G_{4}^{S E}(Z \alpha)$ & $G_{5}^{S E}(Z \alpha)$ \\
\hline 0 & $0.912^{a}$ & & & \\
\hline 5 & $0.860(150)^{b}$ & & & \\
\hline 10 & $0.707(11)^{b}$ & $0.601(38)^{b, c}$ & $0.492(75)^{b, c}$ & $0.412(113)^{b, c}$ \\
\hline 15 & $0.619(1)^{b}$ & & & \\
\hline 20 & $0.530^{b}$ & $0.436(5)^{b, c}$ & $0.349(5)^{b, c}$ & $0.286(9)^{b, c}$ \\
\hline 25 & $0.445^{b}$ & & & \\
\hline 30 & $0.362^{b}$ & $0.284(2)^{b, c}$ & $0.220(2)^{b, c}$ & $0.175(2)^{b, c}$ \\
\hline \multicolumn{5}{|c|}{ Results of the extrapolation over $Z$} \\
\hline 1 & $0.89(2)$ & $0.75(17)$ & $0.62(21)$ & $0.53(27)$ \\
\hline
\end{tabular}

Table 2: Extrapolation over $Z .^{a}$ - from Ref. [15]; ${ }^{b}$ - from Ref. [14]; ${ }^{c}-$ from Refs. [13,14]. 


\begin{tabular}{||c|c|c||}
\hline \hline$n$ & $G_{n}^{S E}(\alpha)$ & $\widetilde{G}_{n}$ \\
\hline 2 & $0.89(02)$ & $3.56(09)$ \\
3 & $0.75(17)$ & $3.38(78)$ \\
4 & $0.62(21)$ & $3.31(114)$ \\
5 & $0.53(27)$ & $3.29(167)$ \\
\hline 6 & $0.46(28)$ & $3.30(200)$ \\
7 & $0.40(31)$ & $3.30(250)$ \\
8 & $0.36(33)$ & $3.30(300)$ \\
9 & $0.33(35)$ & $3.30(350)$ \\
10 & $0.30(36)$ & $3.30(400)$ \\
11 & $0.27(37)$ & $3.30(450)$ \\
12 & $0.25(38)$ & $3.30(500)$ \\
\hline \hline
\end{tabular}

Table 3: Extrapolation over $n$. 


\begin{tabular}{||c|c|c||}
\hline \hline & $\left\langle\Sigma_{1} G_{C} \Sigma_{1}>\right.$ & $<\Sigma_{2}^{l a d}>$ \\
\hline$E(n s)$ & $-\frac{8}{27 \pi^{2}} \frac{1}{n^{3}} m \log ^{3} \frac{1}{(Z \alpha)^{2}}, a$ & 0 \\
\hline$\Delta(n)$ & $\frac{16}{9 \pi^{2}}\left(\log n-\psi(n+1)+\psi(2)-\frac{n-1}{n}\right) \log ^{2} \frac{1}{(Z \alpha)^{2}}, b, c$ & $\frac{4}{9 \pi^{2}} \frac{n^{2}-1}{n^{5}} \log ^{2} \frac{1}{(Z \alpha)^{2}}, d$ \\
\hline$E(n p)$ & 0 & $\frac{4}{27 \pi^{2}} \frac{n^{2}-1}{n^{5}} \log ^{2} \frac{1}{(Z \alpha)^{2}}, d$ \\
\hline$\Gamma(2 p)$ & 0 & $-\frac{2^{10}}{3^{9} \pi} \ln \frac{1}{(Z \alpha)^{2}}\left(-2 \ln \frac{4}{3}+\frac{61}{24}\right), b, e, f, g, h$ \\
\hline$\Gamma(3 s)$ & $-\frac{2^{10}}{5^{9} \pi} \ln \frac{1}{(Z \alpha)^{2}}\left(2 \ln \frac{5}{4}+\frac{387}{40}\right), f, h$ & 0 \\
\hline \hline
\end{tabular}

Table 4: Leading logarithmic contributions of two-loop self-energy in the Yennie gauge to different energy levels and decay widths $(\Gamma)$. Results are done in units of $\alpha^{2}(Z \alpha)^{6} m .^{a}$ - Ref. [23]; ${ }^{b}$ - Ref. [8]; ${ }^{c}-$ Ref. [24]; ${ }^{d}$ - Refs. $[25,26] ;^{e}$ - Ref. [27] (numerically); ${ }^{f}$ - Ref. [28] (numerically); ${ }^{g}$ - Ref. [29] (analytically and numerically) $;^{h}$ - Ref. [30] (analytically). 


\begin{tabular}{||c|c|c|c||}
\hline \hline Ref. & $\delta E(1 s)$ & $\delta E(2 s)$ & $\delta E(1 s) / 8 \delta E(2 s)$ \\
\hline [31 & -7.4 & -0.93 & 1 \\
32 & 2.8 & 0.35 & 1 \\
34 & $-7.1(9)$ & $-0.90(6)$ & $0.99(13)$ \\
\hline \hline
\end{tabular}

Table 5: Recoil correction in order $(Z \alpha)^{6} \mathrm{~m}^{2} / M$ in the hydrogen atom in $\mathrm{kHz}$. Numerical results of Ref. [34] include also higher order in $(Z \alpha)$ corrections. 


\begin{tabular}{||c|c|c|c|c|c|c||}
\hline \hline$n$ & $\Delta^{G}(n)$ & $\Delta^{V P}(n)$ & $\Delta^{I I}(n)$ & $\Delta^{\text {Hyd }}(n)$ & $\Delta^{\text {Deu }}(n)$ & $\Delta^{I s o}(n)$ \\
\hline 2 & $39(1)$ & 8 & $-11(5)$ & $-187232(5)$ & $-187225(5)$ & 7.3 \\
3 & $33(7)$ & 8 & $-15(7)$ & $-235079(10)$ & $-235073(10)$ & 5.9 \\
4 & $27(9)$ & 9 & $-17(8)$ & $-254428(12)$ & $-254423(12)$ & 4.7 \\
5 & $22(11)$ & 8 & $-18(9)$ & $-264162(15)$ & $-264158(15)$ & 4.0 \\
6 & $20(12)$ & 8 & $-19(9)$ & $-269747(15)$ & $-269743(15)$ & 3.5 \\
7 & $17(13)$ & 7 & $-19(10)$ & $-273246(16)$ & $-273243(16)$ & 3.2 \\
8 & $16(14)$ & 7 & $-20(10)$ & $-275583(17)$ & $-275580(17)$ & 3.0 \\
9 & $14(15)$ & 7 & $-20(10)$ & $-277221(18)$ & $-277218(18)$ & 2.9 \\
10 & $13(16)$ & 7 & $-20(10)$ & $-278413(19)$ & $-278410(19)$ & 2.7 \\
11 & $12(16)$ & 7 & $-21(10)$ & $-279308(19)$ & $-279305(19)$ & 2.6 \\
12 & $11(17)$ & 7 & $-21(10)$ & $-279996(20)$ & $-269993(20)$ & 2.5 \\
\hline \hline
\end{tabular}

Table 6: Corrections and results for the difference (1) in the hydrogen and deuterium atoms and isotopic shift. The one-loop self-energy $\left(\Delta^{G}(n)\right)$, the vacuum polarization $\left(\Delta^{V P}(n)\right)$ contribution and the two-loop correction $\Delta^{I I}(n)$ are the same in both atoms. 


\begin{tabular}{||c|c|c|c||}
\hline \hline & \multicolumn{3}{|c||}{ Correction to $n^{3} \cdot\left(\Delta E^{I}(n s)-\Delta E^{I I}(n s)\right)($ in kHz) } \\
\hline$n$ & $(Z \alpha)^{6} m^{2} / M$ & $(Z \alpha)^{4} m^{3} / M^{2}$ & Total \\
\hline 1 & 0 & 26 & 26 \\
2 & 1 & -13 & -12 \\
3 & 2 & -26 & -24 \\
4 & 2 & -33 & -31 \\
5 & 2 & -36 & -35 \\
6 & 2 & -39 & -37 \\
7 & 2 & -41 & -39 \\
8 & 2 & -42 & -41 \\
9 & 2 & -43 & -42 \\
10 & 2 & -44 & -43 \\
11 & 2 & -45 & -43 \\
12 & 2 & -46 & -44 \\
\hline \hline
\end{tabular}

Table 7: Recoil contributions to difference of the definitions. 